\title{
How do Teachers Apply Computational Thinking Activities in Elementary School? From Continuing Education to the classroom
}

Original Title: Como os professores aplicam o Pensamento Computacional em atividades destinadas ao Ensino Fundamental I? Da Formação Continuada para a sala de aula

\author{
Suéllen R. Martinelli ${ }^{12}$, Luciana Ap. M. Zaina ${ }^{2}$, Tiemi C. Sakata ${ }^{2}$ \\ ${ }^{1}$ Mestrado em Ciência da Computação, Universidade Federal de São Carlos (UFSCar) - Sorocaba, SP - Brasil \\ ${ }^{2}$ Programa de Pós-Graduação em Ciência da Computação, Universidade Federal de São Carlos (UFSCar) - Sorocaba, SP - Brasil
}

\begin{tabular}{l} 
ARTICLE INFO \\
\hline Article history: \\
Received 08 February 2019 \\
Accepted 06 April 2020 \\
Available online 05 May 2020
\end{tabular}

Keywords:

Computational Thinking

Primary School

Continuing Education Course

ISSN: $2595-9077$

DOI: JCThink.2019.v3.n1.p50

\section{ABSTRACT}

INTRODUCTION: There is no consensus of what skills can be developed from the learning of Computation Thinking (CT). However, teachers and researchers agreed that by the learning of $C T$ individuals can acquire and expand many abilities. Courses have been carried out in Brazil and in other countries with the aim of motivating teachers on adding CT to classroom practices. OBJECTIVE: This article to present a case study that investigated the feasibility of introducing activities of CT for primary school children. Such activities were created and mediated by the teachers of Primary School. METHOD: First, a continuing education course about Computational Thinking was conducted with the participation of 14 teachers. The participants could understand the fundamentals of CT, and consequently, became able to planning and conducting CT activities by themselves in their classrooms. After, we analyzed the data gathered during the course under the quantitative and qualitative lenses. RESULTS: The results showed that the participants had a degree of agreement between $56 \%$ and $71 \%$ regarding the understanding and applicability of CT to developing skills in Primary School. The qualitative findings revealed that the participants could prepare activities that linked CT to topics presented in the subjects of Primary School. Besides, we could identify three patterns of shaping the activities in which this "computational think" were incorporated. CONCLUSION: Practices to stimulate the CT in students are mediated by using different teaching strategies as such Gamification and Culture Maker. These activities also enable the development of skills related to the National Curricular Common Base.

\section{RESUMO}

INTRODUCÃO: Não há um consenso sobre quais habilidades podem ser desenvolvidas a partir do aprendizado do Pensamento Computacional $(P C)$. No entanto, professores e pesquisadores concordaram que, com o aprendizado do PC, os indivíduos podem adquirir e expandir diversas habilidades. Cursos sobre PC têm sido realizados no Brasil e em outros países com o objetivo de motivar professores à adicionarem o PC em suas práticas de sala de aula. OBJETIVO: Este artigo tem como objetivo apresentar um estudo de caso que investigou a viabilidade da introdução de atividades sobre PC para crianças do Ensino Fundamental I. Tais atividades foram criadas e mediadas pelos próprios professores do Ensino Fundamental I. MÉTODO: Primeiramente, foi realizado um curso de formação continuada sobre Pensamento Computacional com a participação de 14 professores. Os participantes puderam compreender os fundamentos do PC e, consequentemente, tornaram-se capazes de planejar atividades sobre PC e aplicá-las em suas respectivas salas de aula. Depois, foram analisados os dados coletados durante a formação sob as lentes quantitativa e qualitativa. RESULTADOS: Os resultados mostraram que os participantes apresentaram um grau de concordância entre $56 \%$ e $71 \%$ em relação à compreensão e aplicabilidade do PC quanto ao desenvolvimento dessas habilidades no Ensino Fundamental I. Os achados qualitativos revelaram que os participantes puderam preparar atividades de ensino vinculadas ao PC e aos tópicos apresentados nos componentes curriculares do Ensino Fundamental I. Além disso, foram identificados três padrões de formatos de atividades nas quais esse "pensar computacional" foi incorporado. CONCLUSÃO: Práticas que estimulam o PC nos alunos são mediadas pelo uso de diferentes estratégias de ensino como a Gamificação $e$ a Cultura Maker. Essas atividades também possibilitam o desenvolvimento de habilidades relacionadas com a Base Curricular Comum Nacional.

\section{Introdução}

O termo Pensamento Computacional (PC), do inglês Computational Thinking, foi difundido na academia a partir de 2006, por Jeannette Wing (Wing, 2006). A pesquisadora esclarece que é possível utilizar os conceitos e habilidades fundamentais que sustentam a Ciência da Computação para interpretar e resolver problemas de todas as áreas do conhecimento. O PC trata da capacidade de se pensar de forma abstrata, reduzir problemas em partes menores e mais simples de se resolver, sem 
obrigatoriamente ser preciso a aplicação de técnicas de programação ou de meios digitais para esse fim (Wing, 2014).

$\mathrm{Na}$ literatura, não há um consenso entre os pesquisadores que atuam em Computação e Educação sobre quais são as concepções que caracterizam o Raciocínio Computacional (Kalelioglu, Gülbahar \& Kukul, 2016). Porém, estes estão em comum acordo em reconhecer que esse "pensar computacional" precisa ser uma habilidade de todos e para todos. O ensino de conceitos básicos de Computação nas escolas é fundamental, assim como a sua inserção no atual currículo brasileiro por meio de políticas nacionais, possibilitando a formação de cidadãos capazes de viverem em uma sociedade globalizada (Silva, da Silva \& França, 2017). Para isso, é vital promover a formação continuada de professores do Ensino Básico para gerar investigações quanto à disseminação e avaliação das habilidades ligadas ao PC para a sua efetiva aplicação nas instituições de ensino (Valente, 2016).

Este artigo tem como objetivo apresentar um Estudo de Caso (EC) que investigou práticas educativas voltadas ao desenvolvimento do PC em crianças, sendo tais atividades construídas e mediadas por docentes do Ensino Fundamental I (EFI) de instituições públicas. O EC teve como foco verificar se é possível inserir o PC em atividades de ensino direcionadas ao EFI e como esse raciocínio foi conduzido em práticas educacionais pelos professores. Como objetivo secundário, o EC ainda buscou levantar por meio de surveys qual foi a perspectiva dos docentes quanto à compreensão e a aplicabilidade do Raciocínio Computacional em sua realidade escolar. Para esses propósitos, foi realizado um curso de formação continuada para que os pesquisados compreendessem o PC para atuarem no planejamento e condução de atividades que fomentem tal competência nas crianças. A partir dos dados coletados foram conduzidas duas análises, sendo uma quantitativa e baseada na Estatística Descritiva (Lazar, Feng \& Hochheiser, 2010), e a segunda, de cunho qualitativo, foi apoiada na Teoria Fundamentada (Charmaz, 2009). Entre as práticas criadas pelos participantes foram extraídos três formatos (padrões) de atividades de ensino que viabilizam o desenvolvimento de habilidades do PC.

O artigo está estruturado como segue: a Seção 2 apresenta conceitos e trabalhos relacionados ao PC na educação básica; a Seção 3 fornece explicações sobre a metodologia utilizada no Estudo de Caso incluindo as técnicas e procedimentos adotados para a análise de dados; a Seção 4 aborda os resultados pertinentes a avaliação quantitativa; seguido da Seção 5 que trata dos produtos gerados a partir da análise qualitativa; a Seção 6 responde as perguntas de pesquisa por meio da confirmação de hipóteses; e para concluir, a Seção 7 realiza as considerações finais.

\section{Fundamentação Teórica e Trabalhos Relacionados}

Atualmente, o Pensamento Computacional é entendido como um conjunto de habilidades essenciais à formação do indivíduo, para capacitá-lo na resolução de problemas diversificados, sendo seu domínio tão importante quanto ao da leitura, da escrita e da aritmética (Wing, 2014). Percebendo a relevância do tema, países como Austrália, Estados Unidos, Canadá e Reino Unido promovem o ensino de Computação em suas escolas desde o Ensino Básico (França \& Tedesco, 2015). Organizações como a Computer Science Teachers Association (CSTA), a Association for Computing Machinery (ACM) e a International Society for Technology in Education (ISTE) compuseram normativas difundidas pelo mundo e destinadas ao ensino de Computação e do Pensamento Computacional.

Há também a difusão de iniciativas que produzem e distribuem exemplos e materiais didáticos gratuitos sobre como conduzir atividades que estimulem o PC nas 
crianças. Algumas dessas iniciativas são: o projeto britânico Computing At School ou CAS (https://www.computingatschool.org.uk), o CS Unplugged (http://csunplugged.org/) da Nova Zelândia, a plataforma Code.org (https://studio.code.org), e a formação online Exploring Computational Thinking (https://edu.google.com), para docentes, da Google for Education.

A iniciativa Barefoot (https://barefootcas.org.uk) foca na produção de materiais e na realização de treinamentos para os professores do Ensino Fundamental I do Reino Unido. Uma vez que o público-alvo atendido pelo projeto é o mesmo considerado por esta investigação, foram adotadas as definições acerca do PC, estipuladas pelo projeto britânico, sendo essas definidas como: o Raciocínio Lógico, que auxilia a analisar fatos; a ideia de Algoritmo, que exige uma sequência precisa de instruções para executar uma tarefa; a Decomposição o, capacidade de quebrar um problema em partes menores e gerenciáveis; a percepção de Padrões, para ser capaz de reconhecer similaridades entre problemas; a Abstração, habilidade que ajuda na sintetização dos fatos para decidir o que é importante; e a Avaliação, capacidade de julgar com base em fatos, auxiliando na tomada de decisões.

No Brasil, a Sociedade Brasileira de Computação (SBC) também estimula projetos sobre Computação e Pensamento Computacional no Ensino Básico, sendo alguns grupos responsáveis por realizar intervenções, oficinas e cursos com a comunidade. Das iniciativas ${ }^{1}$ mantidas por universidades, destacam-se: o Meninas Digitais, o Computação na Escola, o Laboratório de Inovação Tecnológica na Educação (LITE) e o Computing to You! (C2Y!).

Aliado ao crescente número de pesquisas sobre práticas educacionais direcionadas ao PC no contexto brasileiro (Ferreira et al., 2015; Barcelos, Bortoletto \& Andriolli, 2016; de Melo Reis et al., 2017; Silva, da Silva \& França, 2017; Brum, Konzen \& Schmachtenberg, 2018), a SBC também têm discutido com representantes da Base Nacional Comum Curricular (BNCC), sobre a alteração da Base para adequar um currículo voltado ao desenvolvimento do PC em seu universo e cultura digital. Esse debate é significativo, uma vez que a atual versão da BNCC aborda o PC de forma estrita, integrando-o apenas à Matemática, e não como área do conhecimento, como orienta a SBC (Leite \& da Silva, 2017).

Diante dos desafios e oportunidades apontadas por França \& Tedesco (2015) e Valente (2016) para contribuir na integração de PC no Ensino Básico brasileiro, destacam-se alguns trabalhos relacionados como: a condução de Atividades Desplugadas interdisciplinares sobre Computação, entre alunos do Ensino Fundamental II e Médio (Ferreira et al., 2015); a aplicação de um curso para estudantes do $8^{\circ}$ e $9^{\circ}$ ano com foco no desenvolvimento de jogos eletrônicos, com o uso de abordagens de programação e Storytelling (de Melo Reis et al., 2017); e o uso da Aprendizagem Baseada em Problemas e outras duas abordagens, empregadas em oficinas com alunos entre o $3^{\circ}$ e $8^{\circ}$ ano do Ensino Fundamental, para estimular o PC diante de situações-problema (Brum, Konzen \& Schmachtenberg, 2018). Também há alguns estudos que se enquadram na capacitação docente como a formação inicial e continuada sobre PC, na modalidade de Ensino à Distância, dedicada para professores de Matemática (Barcelos, Bortoletto \& Andriolli, 2016); e a formação presencial sobre PC, ofertada aos docentes de escolas públicas de Recife - PE (Silva, da Silva \& França, 2017).

Esta investigação diferencia-se das citadas acima por oportunizar uma formação continuada entre docentes do EFI, fazendo-os compreender, desenvolver e levar para a realidade educacional de cada professor o Pensamento Computacional. Além disso, os resultados deste estudo são suportados por uma avaliação qualitativa, sendo essa uma

1 Para conhecer essas e outras iniciativas, acesse: http://www.sbc.org.br/institucional-3/chancela-sbc/. 
técnica pouco usada em investigações direcionadas à Computação. Assim, espera-se que esta pesquisa possa em aspectos respectivos ao planejamento e condução de atividades de ensino dos envolvidos, adaptadas com a intenção de estimularem o PC nos alunos.

\section{Metodologia do Estudo de Caso}

Por não existir obrigatoriedade na implantação de currículos específicos ou, pelo menos, de práticas que estimulem o PC, essas atividades acabam acontecendo somente por mediação de um profissional da Computação, quando a instituição possui o mesmo. Ao considerar esse contexto e a infraestruturas das escolas no país, tem-se como motivação a tentativa de capacitar o próprio docente do Ensino Básico, para que atue como elaborador e mediador de atividades educacionais sobre PC, de acordo com a sua realidade institucional. Assim, esta pesquisa se propõe a investigar as seguintes questões:

Pergunta 1 (P1): Como é possivel incluir o Pensamento Computacional em Atividades de Ensino direcionadas ao Ensino Fundamental I?

Pergunta 2 (P2): Como o docente trata/conduz as habilidades do Pensamento Computacional em Atividades de Ensino direcionadas ao Ensino Fundamental I?

Para responder as perguntas foi planejado e conduzido um Estudo de Caso de caráter exploratório, ou seja, uma investigação que favorece a compreensão inicial sobre um determinado problema ou situação desconhecida pelo pesquisador, a fim de levantar métricas e gerar hipóteses para novas investigações (Lazar, Feng \& Hochheiser, 2010). Os dados coletados foram analisados de forma quantitativa e qualitativa. A estrutura do EC efetuado obedece a descrição da Figura 1, organizado em quatro fases.

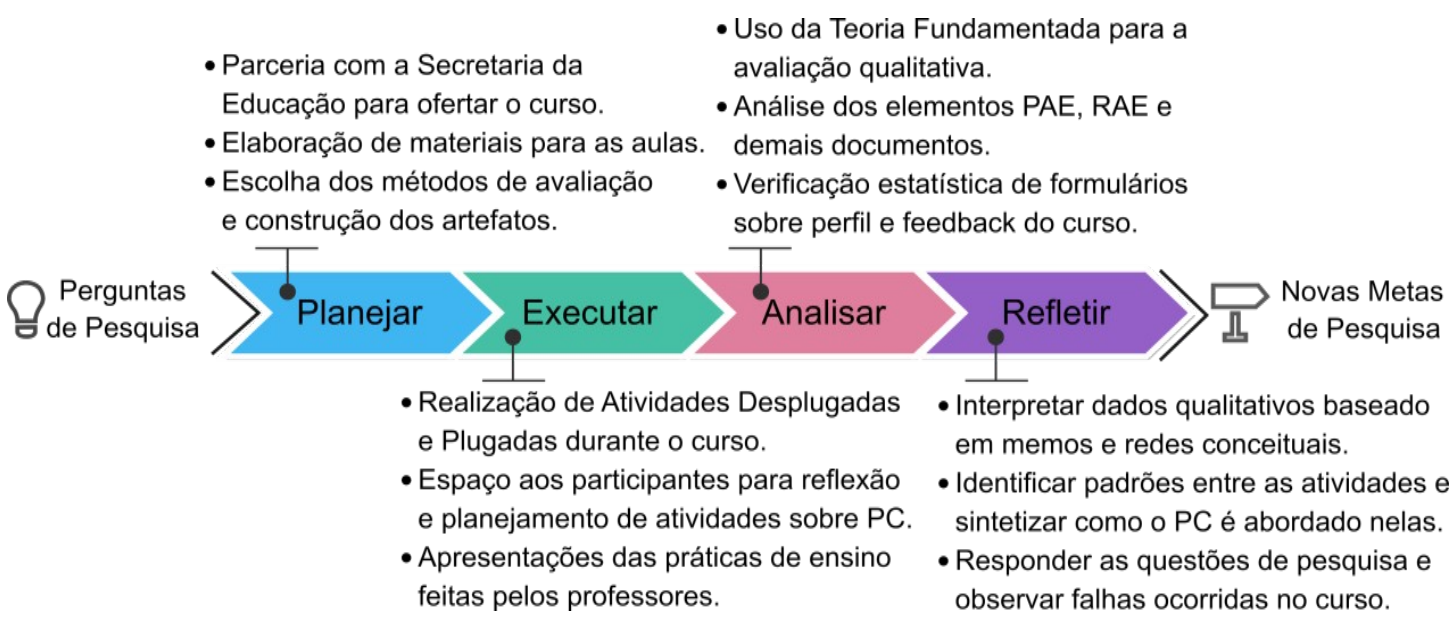

Figura 1. Visão geral da metodologia: métodos, artefatos e ações.

A fase de planejamento teve o objetivo de definir uma formação continuada sobre PC para profissionais do EFI, incluindo a elaboração de materiais e métodos de avaliação dos artefatos propostos. Nesta fase, foram concebidas apresentações eletrônicas para as aulas e artefatos destinados à coleta de dados ${ }^{2}$.

Para viabilizar a execução, foi firmada uma parceria entre a UFSCar Sorocaba e a Secretaria de Educação de Salto de Pirapora. A Figura 2 trata da organização do curso ministrado, que organiza o que foi aplicado em cada aula e as habilidades do PC

2 Alguns dos materiais desenvolvidos e artefatos aplicados na formação estão disponíveis em: http://bit.ly/MateriaisFormacaoPC. 
abordadas. Com duração de 120 minutos cada, as aulas foram ministradas em encontros semanais e/ou quinzenais, alocadas de acordo com a disponibilidade dos participantes. Todas as aulas aconteceram na EMEF Prof. Roberto Marcello, durante o primeiro semestre de 2017.

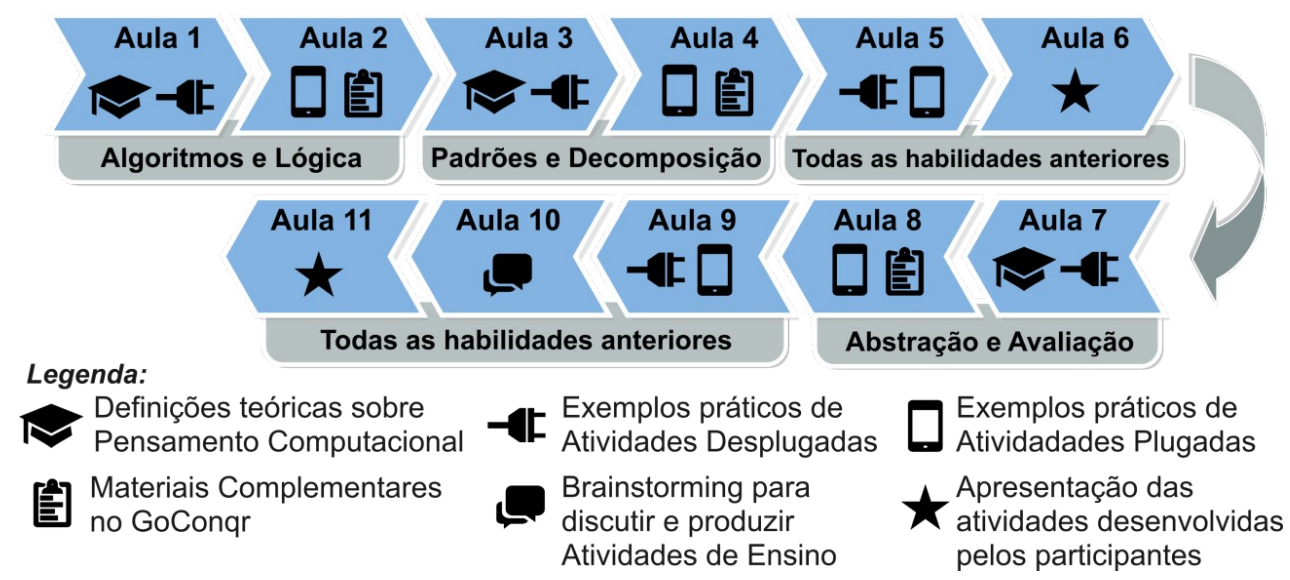

Figura 2. Aulas e organização dos conceitos de PC abordados na Formação Continuada.

Durante a fase de execução os conceitos do PC foram introduzidos em pares e de forma incremental, por meio de atividades relacionadas a assuntos de vários componentes curriculares. Entre as práticas conduzidas com os professores, destacam-se as Atividades Desplugadas, caracterizadas por não usarem nenhum meio digital para abordar o PC, e as Atividades Plugadas, realizadas no laboratório de informática da instituição em que o curso foi aplicado. Além dos computadores usados nas Atividades Plugadas, foram empregados nessas práticas 9 tablets emprestados pelo Instituto Federal de Educação, Ciência e Tecnologia de São Paulo (IFSP Câmpus Itapetininga).

A formação iniciou com 41 docentes do EFI da rede municipal de educação de Salto de Pirapora-SP, sendo que desses, 14 concluíram o curso. Os dados considerados neste estudo são apenas dos professores que finalizaram o curso, tendo a maioria deles idade entre 31 e 40 anos, com presença predominante de profissionais do sexo feminino. Aos 54,5\% dos participantes, o tempo de experiência é entre 6 e 10 anos de docência, e 63,5\% elencaram ter mais dificuldade em lecionar temas da disciplina de Matemática.

As atividades de ensino sobre PC foram produzidas pelos docentes em duplas ou individualmente, em que estes escolheram livremente um assunto de um componente curricular a fim de estimular o Raciocínio Computacional nas crianças em suas práticas de ensino, por meio de um assunto ou conceito chave. Essas práticas foram documentadas em dois momentos: antes da condução, na Planilha de Dados Primários (PAE) para descrever aspectos quanto ao planejamento da atividade; e após a condução, no Roteiro da Atividade de Ensino (RAE) para documentar os procedimentos adotados na prática desenvolvida e pontuar as relações observadas entre a atividade feita e as habilidades do PC estimuladas. Uma vez que esses processos foram feitos pelos docentes para a construção de duas práticas de ensino, cada dupla de arquivos PAE e RAE foram preenchidos antes das aulas 06 e 11, sendo essas respectivas as apresentações das práticas criadas. As atividades documentadas pelos professores foram aplicadas por eles em suas próprias salas de aula. A Figura 3 ilustra nas três primeiras imagens o envolvimento dos professores em atividades exemplificadas durante a formação, enquanto que as fotos inferiores retratam momentos de diferentes práticas de ensino aplicadas pelos docentes em escolas do EFI. 


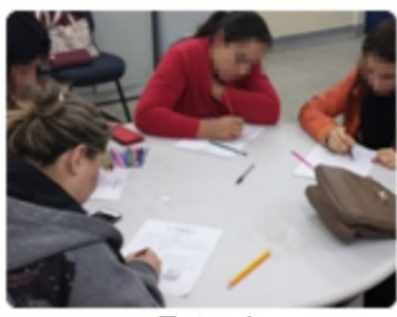

Foto $\mathrm{A}$

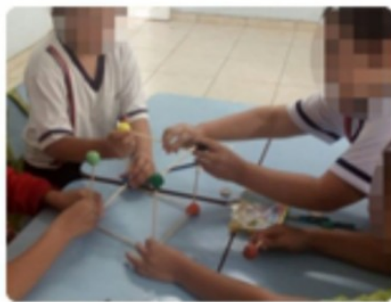

Foto D

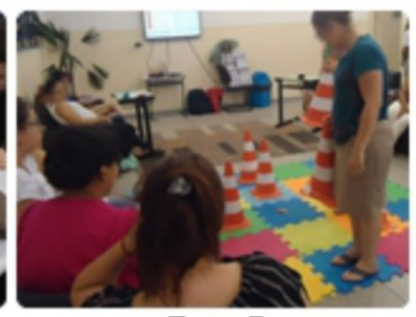

Foto B

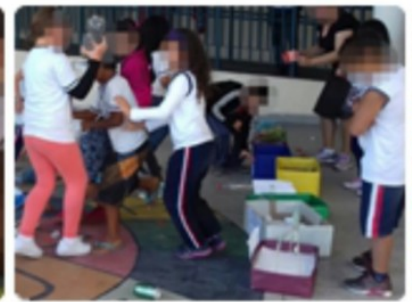

Foto $\mathrm{E}$

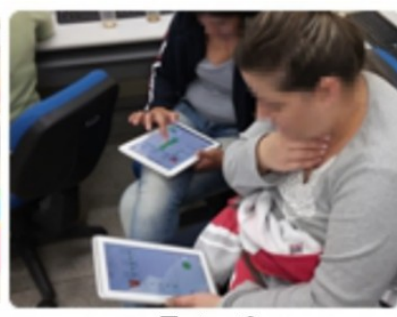

Foto C

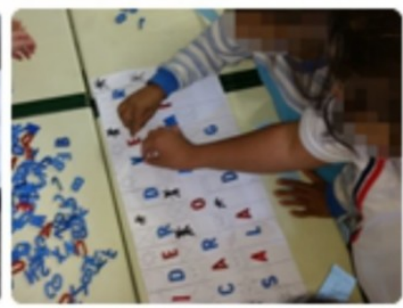

Foto $\mathrm{F}$

Figura 3. Docentes em Atividades Desplugadas (Foto A e B) e em Atividade Plugada (Foto C). Práticas aplicadas com temas relacionados à Matemática (Foto D), Ciências (Foto E) e Língua Portuguesa (Foto F).

A fase de análise teve como finalidade o tratamento e avaliação dos dados capturados na formação, referentes a objetivos e métodos distintos, utilizando-se da Estatística Descritiva para a análise quantitativa (Lazar, Feng \& Hochheiser, 2010) e da Teoria Fundamentada para a análise qualitativa (Charmaz, 2009). As técnicas e procedimentos envolvidos na avaliação dos dados obtidos por meio dos artefatos AHPC, PAE e RAE são especificados nas Seções 3.1 e 3.2.

A fase de reflexão pertinente ao $\mathrm{EC}$, focou na interpretação dos dados e na resolução das perguntas de pesquisa que nortearam esta investigação. Os conteúdos e resultados que equivalem a essa etapa são abordados na Seção 4, Seção 5 e Seção 6.

\subsection{Técnicas e Procedimentos da Análise Quantitativa}

Para a avaliação quantitativa foi considerada a Estatística Descritiva, a fím de avaliar dados não probabilísticos, capturados a partir de questões fechadas, para obter resultados percentuais (Lazar, Feng \& Hochheiser, 2010) que complementem as respostas pertinentes ao objetivo primário deste estudo.

A Estatística Descritiva foi usada nos dados capturados pelo AHPC (Avaliação de Habilidades do Pensamento Computacional). Esse instrumento foi aplicado em três versões distintas entre os participantes da formação. A aplicação desse survey teve como finalidade obter uma percepção sobre a visão dos pesquisados quanto à sua compreensão e aplicabilidade das habilidades do PC em seus respectivos ambientes de ensino. Cada avaliação AHPC foi empregada ao final de cada conjunto de aulas relacionadas a uma dupla de habilidades do PC, isto é, ao término das aulas 02, 04 e 08 (Figura 2).

As respostas às afirmativas presentes no AHPC foram dadas pelos pesquisados por meio da escolha de uma opção em uma escala likert (Likert, 1932) de seis pontos, com itens entre 'Discordo Totalmente' (extremidade esquerda com peso 1) até 'Concordo Totalmente' (extremidade direita com peso 6). Para cada afirmativa ou questão foi aplicado o padrão de cálculo presente no Quadro 1, a fim de obter o grau de concordância, ou seja, um valor em percentual quanto os participantes concordam sobre uma dada afirmativa ou um conjunto de questões (categoria). $\mathrm{O}$ valor obtido pelo grau de concordância envolve a atribuição de pesos para a quantidade de respostas dadas por 
cada item selecionado na escala, para obter um percentual do quanto os participantes concordam com a respectiva premissa (Choma et al., 2015).

\begin{tabular}{|c|c|c|c|c|}
\hline $\begin{array}{c}\text { Faixa de } \\
\text { Concordância }\end{array}$ & Item da Escala Likert & $\begin{array}{c}N^{o} \text { de } \\
\text { Indivíduos }\end{array}$ & Peso & Total por Item \\
\hline $100 \%$ & Concordo Totalmente & $n_{6}$ & 6 & $n_{6}{ }^{*} 6$ \\
\hline $99 \%$ a $70 \%$ & Concordo Amplamente & $n_{5}$ & 5 & $n_{5} * 5$ \\
\hline $69 \%$ a $51 \%$ & Concordo Parcialmente & $n_{4}$ & 4 & $n_{4}^{*} 4$ \\
\hline $50 \%$ a $31 \%$ & Discordo Parcialmente & $n_{3}$ & 3 & $n_{3} * 3$ \\
\hline $30 \%$ a $1 \%$ & Discordo Amplamente & $n_{2}$ & 2 & $n_{2} * 2$ \\
\hline \multirow[t]{3}{*}{$0 \%$} & Discordo Totalmente & $n_{1}$ & 1 & $n_{1} * 1$ \\
\hline & Total de Individuos: & $\mathrm{N}=\sum_{i=1}^{6} n_{i}$ & $\begin{array}{l}\text { Total por } \\
\text { Item: }\end{array}$ & $\mathrm{S}=\sum_{i=1}^{6} n_{i} * \lambda$ \\
\hline & Nive & de Aceitação da & Pergunta: & $A=S / N$ \\
\hline \multicolumn{5}{|c|}{ Quantidade de perguntas da categoria: } \\
\hline \multicolumn{2}{|c|}{$\begin{array}{r}\text { Soma dos níveis de aceitação das } \\
\text { perguntas da categoria: }\end{array}$} & $\mathrm{C}=\sum_{i=1}^{p} A_{i}$ & & \\
\hline \multicolumn{2}{|c|}{ Nivel de aceitação da categoria: } & $(C / 6 p) * 100$ & & \\
\hline
\end{tabular}

Quadro 1. Padrão de cálculo aplicado na análise quantitativa. Adaptado de Lazar, Feng e Hochheiser (2010) e Choma et al. (2015).

\subsection{Técnicas e Procedimentos da Análise Qualitativa}

A análise qualitativa foi guiada pelo método da Teoria Fundamentada, em inglês, Grounded Theory. A intenção desse tipo de investigação é gerar uma pesquisa fundamentada nos dados coletados junto aos participantes que experimentaram de um determinado processo e, assim, conceber ou descobrir uma teoria. A técnica exige que o pesquisador aprofunde-se na análise por meio de sucessivas comparações entre os dados. Os resultados vão se solidificado gradualmente até concretizar categorias e conceitos capazes de explicar uma teoria ou modelo (Charmaz, 2009). A Teoria Fundamentada é reconhecida por envolver três níveis de codificação de dados, sendo usados neste estudo com o intuito de reunir dados por similaridades para obter códigos e categorias que expliquem padrões quanto à inserção do PC nas práticas de ensino avaliadas.

$\mathrm{Na}$ Codificação Inicial (Nível 1) foi usada a Codificação Aberta, processo em que são examinados os dados e criados rótulos com base em fragmentos dos dados, para compor códigos significativos aos propósitos de pesquisa. Não se utiliza um conjunto pré-definido de códigos, pelo contrário, os códigos emergem dos dados empíricos. Considerou-se nesse processo todos os artefatos preenchidos pelos participantes, sendo os 26 documentos de registro das atividades PAE e o RAE, além de outros materiais fornecidos pelos docentes, como fotografias e testes aplicados com os alunos sobre as práticas conduzidas. O segundo processo desse nível, a Codificação Focalizada, focou em sintetizar e explicar segmentos maiores de dados, reconhecendo os rótulos mais relevantes e/ou frequentes, capazes de permitirem uma compreensão analítica mais precisa (Charmaz, 2009, p.87).

Na Codificação Axial (Nível 2) foi aplicada a Comparação Incidente por Incidente, que consiste em realizar uma comparação teórica para buscar possíveis correlações entre casos (Charmaz, 2009, p.80), sendo tais casos respectivos às atividades de ensino geradas 
pelos docentes. Para o processo de comparação, esses casos foram comparados também com as anotações do pesquisador e os seguintes documentos listados: i) a BNCC (na versão preliminar) (BNCC, 2017); ii) o CSTA K-12 Computer Science Standards e o Computational Thinking Leadership Toolkit (First Edition), ambos da CSTA; iii) o The Computational Thinker: Concepts and Approaches, da Barefoot; e iv) o Computação na Educação Básica, da SBC. As comparações resultaram em redes conceituais que fundamentam os principais padrões e ocorrências que fazem parte das atividades de ensino sobre PC, sendo uma dessas redes discutida na Seção 5.

Por fim, na Codificação Seletiva (Nível 3), realizou-se uma Amostragem Teórica para entrelaçar novamente a história fragmentada, de maneira lógica e explicativa (Charmaz, 2009, p. 94), com o objetivo de enquadrar cada atividade em uma ou mais proposições (afirmações). Essas proposições retratam padrões identificados nas atividades de ensino e nos demais documentos analisados, demarcando rótulos ou sentenças de agrupamentos desses casos, dando base às respostas das questões de pesquisa. Por último, efetuou-se o Refinamento da Teoria, que possibilita inferir um esquema teórico principal sobre os dados avaliados (Charmaz, 2009). Essa etapa incluiu a conexão de hipóteses junto às perguntas de pesquisa e a construção de modelos gráficos dos formatos das atividades emergentes, abordados na Seção 5.

Os procedimentos descritos desprenderam, em média, em torno de 15 horas semanais, em um período de 5 meses. Toda a análise foi mediada pelo software Atlas.ti ${ }^{3}$, sendo obtido e/ou reunido os seguintes elementos durante a avaliação qualitativa: i) 57 Documentos Primários - materiais PAE, RAE, fotos fornecidas pelos docentes e normas sobre o ensino do PC; ii) 77 Códigos - agrupamentos de dados aplicados pelo pesquisado; iii) 43 Notas Analíticas - memorandos e comentários que descrevem o histórico de interpretação e fundamentam as conexões entre os resultados; iv) 7 Categorias - derivado de comparações entre códigos e notas analíticas, que constitui um conceito e possibilita explicar um dado padrão localizado nas atividades; e v) 1 Relatório Técnico - constituição de um relatório analítico sobre todos os resultados concretizado no EC.

\section{Resultados Quantitativos}

De cada versão do AHPC aplicado obteve-se respostas de 14 participantes no questionário dedicado à Algoritmos e Lógica, 10 respostas no survey sobre Decomposição e Padrões e outras 11 respostas dos pesquisados sobre as habilidades de Abstração e Avaliação. A análise dos dados considerou apenas dados dos participantes que permaneceram até o final do curso, sendo que nem todos os pesquisados estavam presentes quando o AHPC foi aplicado.

As avaliações AHPC foram compostas por seis afirmações e divididas em duas categorias, nomeadas como Compreensão das Habilidades e Aplicabilidade das Habilidades. Na categoria Compreensão das Habilidades, as afirmações respondidas pelos participantes foram: A1) Foi fácil compreender as práticas propostas baseadas nas habilidades de $X$ e $Y, \mathbf{A 2}$ ) Considero fácil lembrar em como usar os conceitos de $X$ e $Y$, e A3) Identifiquei facilmente as habilidades de $X$ e $Y$ no processo de desenvolvimento das atividades de ensino. Os elementos $X$ e $Y$ são classificadas como variáveis nas respectivas sentenças e são correspondentes a dupla de habilidades avaliadas. O gráfico da Figura 4 distribui em escala likert às respostas obtidas e mostra o grau de concordância por dupla de habilidades.

3 O Atlas.ti é um Software de Análise de Dados Qualitativos (SADQ), disponível em: https://atlasti.com/. 


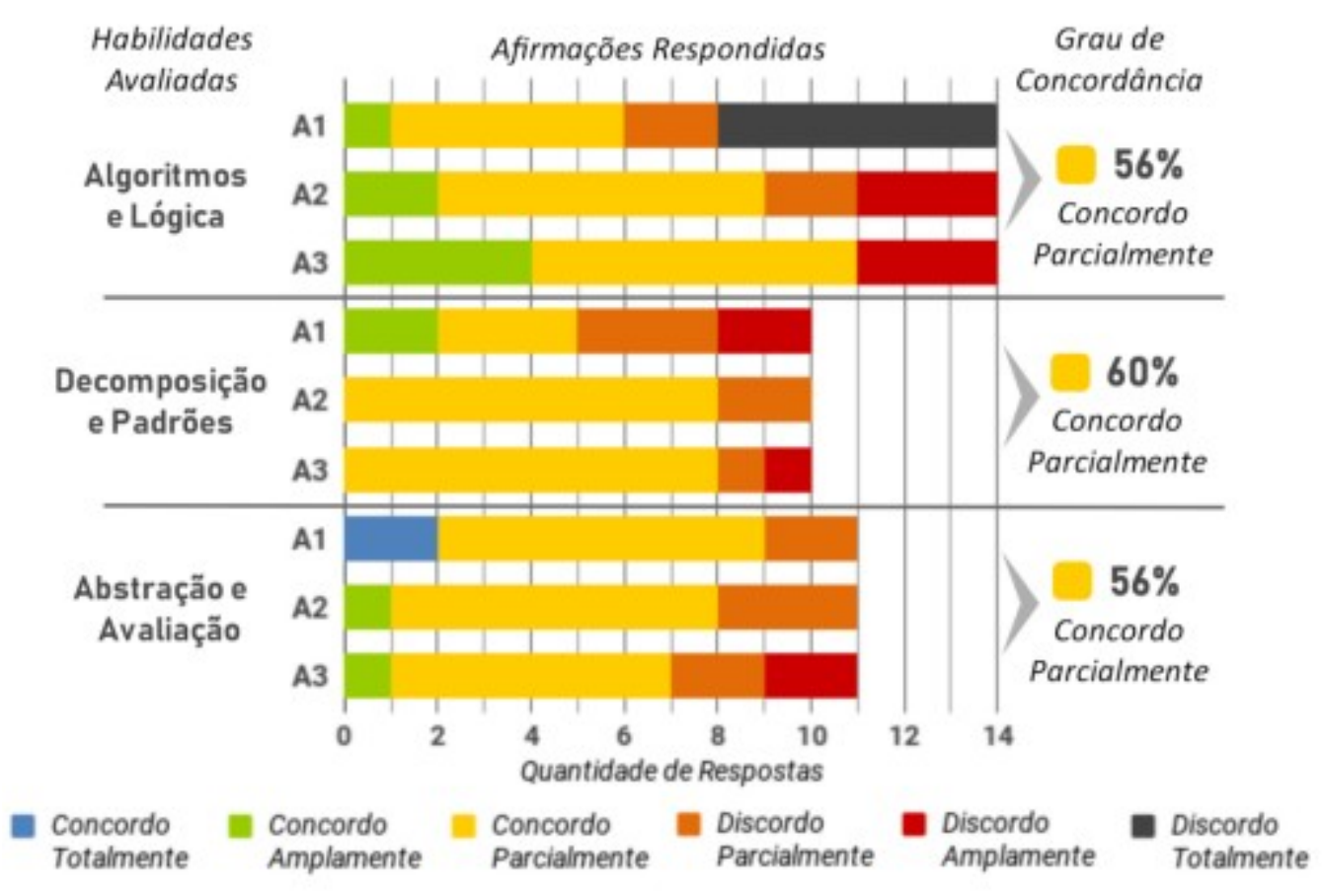

Figura 4. Categoria Compreensão das Habilidades do AHPC avaliada.

Ao comparar os resultados respectivos à afirmação A1, Algoritmos e Lógica tiveram as respostas mais negativas ao apresentar que os pesquisados não compreenderam com facilidade as práticas de ensino baseadas nessas concepções. Todavia, ao observar a mesma afirmativa avaliada entre os três surveys aplicados, há uma diminuição de respostas de extremidade negativa e um aumento das respostas relativas ao centro e à extremidade positiva da escala. Nas demais afirmativas ( $A 2$ e $A 3$ ) há uma persistência em respostas do tipo Concordo Parcialmente, indicando pouca variação nos questionários aplicados entre os diferentes momentos do curso. Essa concentração de respostas fez com que cada grupo de habilidades apresentasse o mesmo grau de concordância, com percentuais aproximados. Desse modo, os dados apontam que a compreensão dos participantes em relação as habilidades de PC mantiveram-se em torno de $56 \%$ e $60 \%$, com concordância parcial.

O segundo gráfico, dado na Figura 5, exibe uma distribuição em escala likert das respostas obtidas e o percentual do grau de concordância por dupla de habilidades avaliadas na categoria Aplicabilidade das Habilidades. As afirmações desse grupo são dadas como: A4) Aplicaria atividades de ensino sobre as habilidades $X$ e $Y$ com frequência, A5) Considero o estímulo aos conceitos de $X$ e $Y$ necessários para a formação do meu aluno, e A6) Identifico facilmente os conceitos de $X$ e $Y$ na preparação de atividades aos meus alunos. Os elementos $X$ e $Y$ permanecem como variáveis nas sentenças, equivalentes a cada dupla de habilidades avaliadas. 


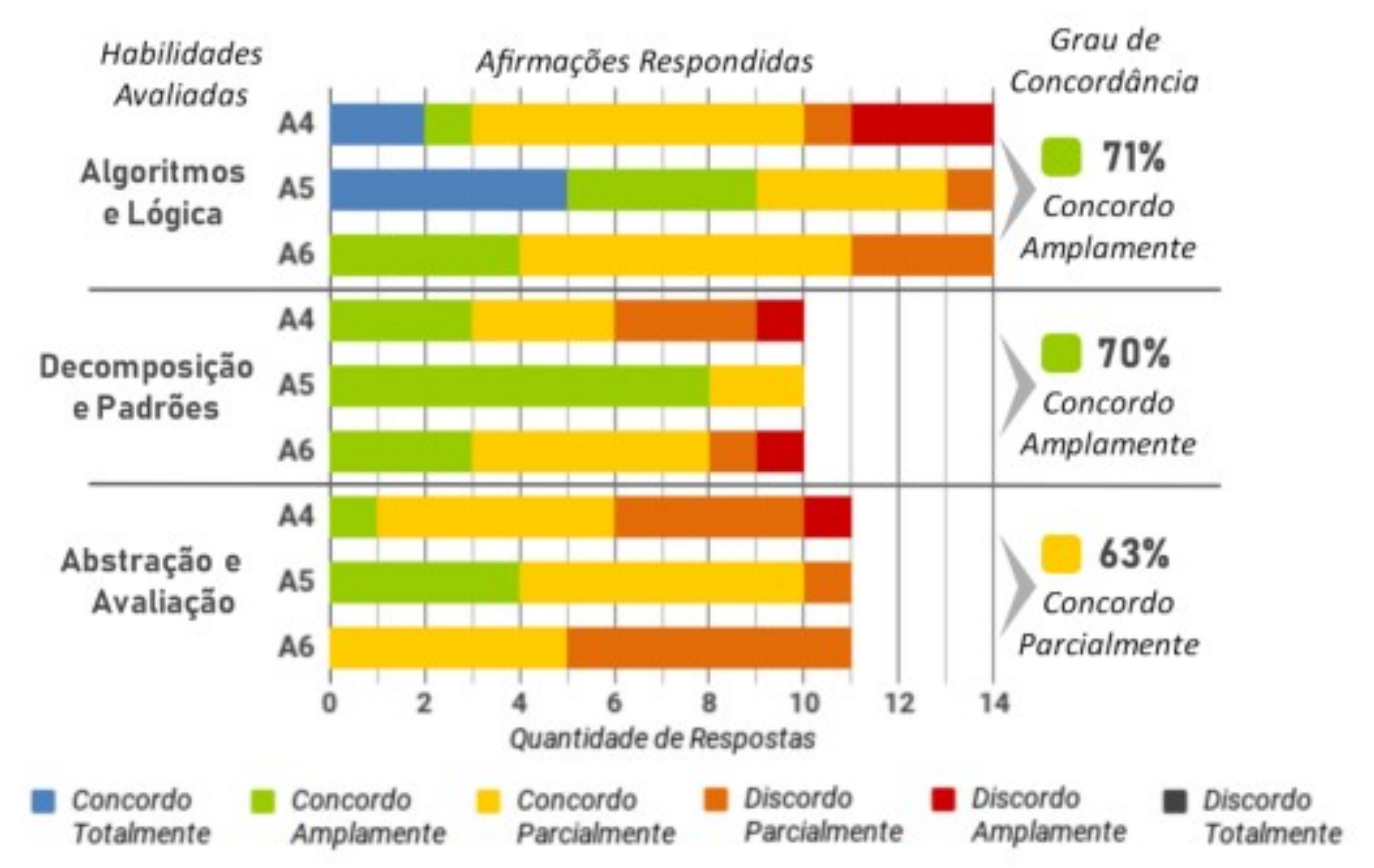

Figura 5. Categoria Aplicabilidade das Habilidades do AHPC avaliada.

A avaliação da categoria Aplicabilidade das Habilidades apresenta uma concentração de respostas entre Concordo Amplamente e Concordo Parcialmente por afirmação. Os resultados do grupo atual mostram-se superiores ao da categoria de Compreensão das Habilidades (Figura 4), uma vez que a percepção dos participantes sobre a relevância e a identificação das habilidades de PC em suas atuais práticas de ensino obtiveram percentuais entre $63 \%$ e $71 \%$, com concordância ampla e parcial. Ao analisar as afirmações por conjunto de habilidades, percebe-se a maior incidência em respostas de concordância na dupla Algoritmos e Lógica, permitindo inferir que, aos participantes, essas habilidades possuem maior viabilidade de serem estimuladas e aplicadas nas suas práticas de ensino. $O$ mesmo acontece com a $A 5$, sentença que apresenta também a maior frequência de respostas de concordância.

Uma reflexão sobre os resultados da análise quantitativa é observado quanto a diferença de concordância entre o entendimento do Raciocínio Computacional por parte dos docentes, e a percepção de aplicabilidade dessa mesma competência nas práticas de ensino. Houve um aumento de aproximadamente $10 \%$ em cada dupla de habilidades avaliadas entre as duas categorias. Isso possibilita inferir que apesar dos participantes terem dificuldades em compreender conceitualmente o que é cada habilidade do PC, estes conseguem visualizar a relevância desses em suas práticas de ensino e na construção do pensamento analítico-lógico das crianças. Nessa perspectiva, vê-se a importância da formação conduzida, a partir de uma perspectiva teórico-prática, uma vez que os pesquisados estudam concepções e possibilidades de atividades sobre PC, mas também aplicam em sala de aulas práticas relacionadas a essa competência e adquirem vivências sobre como estimular essas habilidades nos alunos.

\section{Resultados Qualitativos}

Diante dos procedimentos qualitativos de análise descritos na Seção 3.2, um dos resultados obtidos refere-se aos mapas conceituais, que ilustram conexões entre códigos e que viabilizam uma explicação ou fundamentação sobre uma teoria emergente. $O$ 
mapa conceitual da Figura 6 apresenta uma síntese das principais categorias e conceitos, sendo estas, unidades elementares identificadas quanto à inclusão e condução do PC nas práticas de ensino desenvolvidas.

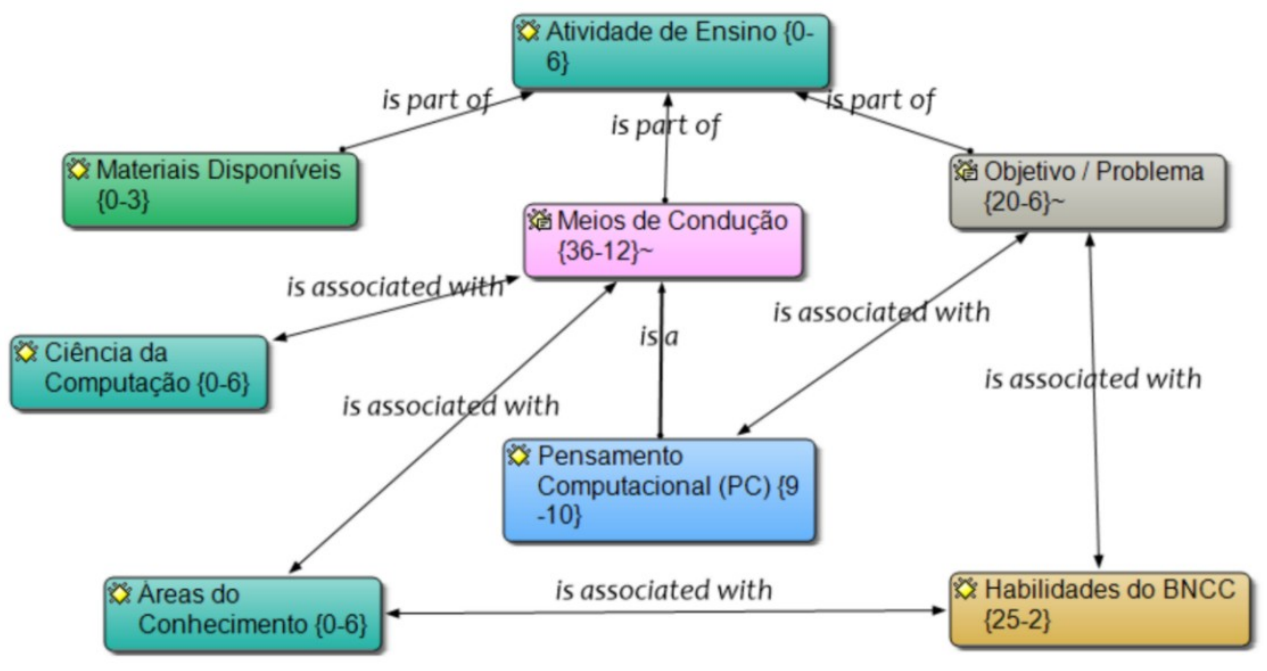

Figura 6. Mapa conceitual com unidades elementares sobre a inclusão e condução do PC nas práticas de ensino.

O mapa conceitual exibe subcategorias relacionadas à primeira categoria Atividade de Ensino, agente que promove um contexto de ensino-aprendizagem baseado em um problema a ser resolvido, constituído por três elementos essências: o Objetivo / Problema, que reúne habilidades da BNCC e do Pensamento Computacional; os Materiais Disponíveis, que expressam insumos e recursos aplicados pelos docentes nas atividades; e os Meios de Condução, que engloba abordagens pedagógicas e procedimentos sobre como as práticas foram conduzidas e/ou mediadas. Em Áreas do Conhecimento há a concatenação por área de conhecimento dos temas e habilidades identificadas nas atividades. Essas habilidades são agrupadas no código Habilidades da $B N C C$, que reúnem 18 habilidades detectadas, sendo elas agregadas aos componentes curriculares de Língua Portuguesa, Artes, Matemática, Ciências e Geografia.

Esse resultado difere do que é exposto pela BNCC, que fixa a relação do PC com ao área do conhecimento de Matemática (BNCC, 2017), ao mostrar que o PC pode ser assimilado em atividades educacionais que perpassam por todas as área do conhecimento. O mapa também expõe a categoria sobre Pensamento Computacional, pontuando a identificação dessa competência nas práticas junto de temas/assuntos específicos da área de Computação (Ciência da Computação), que atuaram estritamente como um dos Meios de Condução para engajar os alunos durante as atividades. O PC também exibe uma relação direta com o Objetivo / Problema, retratando a necessidade de se desenvolver e/ou instigar que o aluno empregue de habilidades do Raciocínio Computacional, bem como da BNCC, para solucionar a problemática ou atingir um objetivo determinado pela atividade.

Embasado nos argumentos da rede conceitual, a Figura 7 ilustra os três Formatos de Atividades de Ensino (FAE) identificados a partir das 13 práticas de ensino (equivalentes aos 26 documentos distribuídos entre PAE e RAE) analisadas por meio da Teoria Fundamentada. Pode-se notar que o PC está presente de duas formas distintas nas FAEs: na área interna, quando é trabalhado junto ao objetivo (problema) da atividade; e na seta externa, quando é usado como método nas atividades. A leitura completa dos diagramas também devem seguir essa mesma lógica, ou seja, ser 
interpretada a partir dos elementos internos, dados pelos círculos, seguidos dos elementos externos, representados por setas e que tratam de todos os métodos ou processos considerados pelos docentes na condução das atividades.
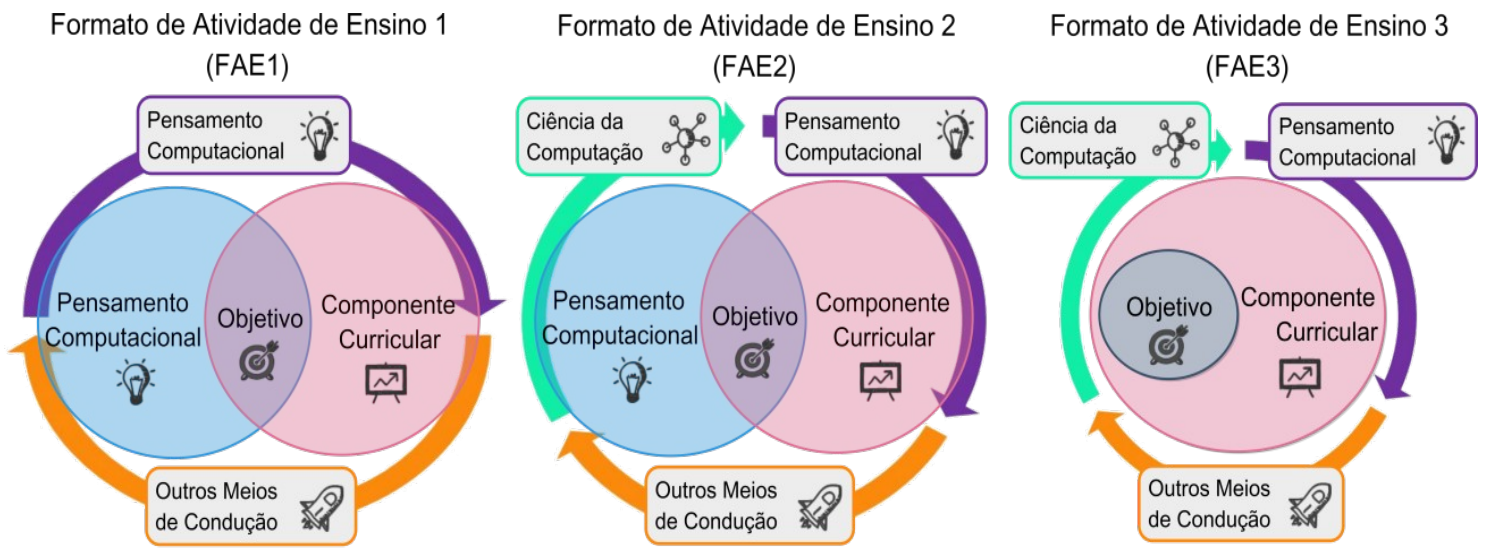

Figura 7. Diagramas dos Formatos das Atividades de Ensino identificados.

Como reflexão sobre os resultados obtidos na análise qualitativa, centrado no tratamento do PC nos padrões FAEs, estes sugerem que os professores utilizaram o PC como método em todas as atividades de ensino, sempre com apoio de outros meios de condução. Além disso, o alvo/objetivo das atividades de ensino abordam sempre um componente curricular como parte da problemática, mas nem sempre houve $\mathrm{o}$ desenvolvimento ou a aplicabilidade de alguma habilidade do PC, por parte da criança, para alcançar esse objetivo. Nota-se também que no FAE2 e no FAE3 as atividades aplicaram um terceiro meio de condução relacionado aos temas/assuntos da Ciência da Computação. Atividades que utilizaram de um tema ou assunto derivado da Computação não tiveram como pretensão a avaliação desse conteúdo, sendo somente incorporada como uma abordagem para desenvolver o Raciocínio Computacional e estimular o interesse dos discentes. Outros detalhes da análise e resultados obtidos encontra-se no relatório técnico de Martinelli, Zaina e Sakata (2018).

\section{Respondendo as Perguntas de Pesquisa}

Para responder cada questão de pesquisa, foram delineadas duas hipóteses ( $H 1$ e H2), sendo a primeira baseada nas características mais frequentes identificadas nas práticas de ensino, enquanto que a segunda aponta para particularidades de menor incidência nas atividades. A obtenção de tais hipóteses em ambas as questões apoiaram-se também em duas categorias principais reconhecidas no mapa conceitual da Figura 6, sendo eles o Pensamento Computacional e o Meios de Condução.

A confirmação de cada hipótese foi feita pela correlação de existência em um ou mais Formatos de Atividades de Ensino (FAE) apresentados na Figura 7. O Quadro 2 indica quais são as FAEs que atendem a cada uma das hipóteses. Esses resultados mostram que, enquanto existe a presença do PC como mecanismo de condução em todos os formatos, apenas os formatos FAE1 e FAE2 abordaram essa competência de maneira integrada a um componente curricular. Por outro lado, todos os casos dependeram de um segundo elemento de conduta da atividade, como destacado na $H 1$ da P2. Esses Outros Meios de Condução são caracterizados por artefatos, insumos, 
procedimentos e/ou estratégias usados(as) nas práticas, como a Gamificação ${ }^{4}$ e a Cultura Maker 5 .

\section{FAE1 FAE2 FAE3}

\begin{tabular}{|c|c|c|c|c|}
\hline \multirow{2}{*}{ P1 } & $\begin{array}{l}\text { H1: O Pensamento Computacional é aplicado } \\
\text { como método e objetivo na Atividade de Ensino. }\end{array}$ & $\checkmark$ & $\checkmark$ & \\
\hline & $\begin{array}{l}\text { H2: O Pensamento Computacional é aplicado } \\
\text { somente como método da Atividade de Ensino. }\end{array}$ & & & $\checkmark$ \\
\hline \multirow{2}{*}{ P2 } & $\begin{array}{l}\text { H1: O Pensamento Computacional é tratado como } \\
\text { método da Atividade de Ensino e vinculado a } \\
\text { outros meios de condução incorporados à prática. }\end{array}$ & $\checkmark$ & $\checkmark$ & $\checkmark$ \\
\hline & $\begin{array}{l}\text { H2: O Pensamento Computacional é tratado como } \\
\text { método da Atividade de Ensino e pode ser } \\
\text { vinculado aos temas da Ciência da Computação. }\end{array}$ & & $\checkmark$ & $\checkmark$ \\
\hline
\end{tabular}

Quadro 2. Hipóteses pertinentes às perguntas de pesquisa, relacionadas com as características identificadas em cada FAE.

\section{Considerações Finais}

A oferta de formações continuadas que esclareçam como incorporar o Raciocínio Computacional de maneira interdisciplinar nas práticas de ensino, são ações que ainda dependem, em maior número, das universidades. Cursos direcionados a essa temática e em diferentes formatos, como presencial (Silva, da Silva \& França, 2017), na modalidade EAD (Barcelos, Bortoletto \& Andriolli, 2016) ou semipresencial, necessitam de abordagens práticas para envolver positivamente os docentes e, em especial, fazerem parte de intervenções constantes do governo.

Este artigo apresentou um Estudo de Caso sobre como é possível inserir o Pensamento Computacional em atividades de ensino por parte dos professores do EFI. Entre os resultados quantitativos, apesar da evasão ocorrida, a compreensão e a aplicabilidade das habilidades do PC, indicaram uma concordância com percentual em torno de $56 \%$ e $71 \%$, de acordo com a avaliação AHPC dos professores concluintes. Já nos resultados da análise qualitativa conclui-se que mesmo com dificuldades desses profissionais (como o desconhecimento sobre PC e aspectos de infraestrutura das escolas), eles conseguiram elaborar e aplicar práticas que estimulassem essa competência em seus discentes, de acordo com a sua realidade institucional. O processo de aprendizagem dessa competência é desafiador em vários aspectos, tanto da perspectiva do professor quanto do aluno, aos quais incluem a escolha de abordagens, técnicas e materiais a serem utilizados neste processo (Bastos et al., 2017), sendo visível nos resultados desse estudo de caso, respostas que atendam a essas variáveis.

Dos Formatos de Atividade de Ensino identificados, foi possível reconhecer que o PC, apoiado em alguma outra estratégia, é facilmente inserido como meio de condução nas práticas de ensino. Quando o PC é um dos objetivos da atividade, é necessário que o professor tenha segurança na compreensão desses conceitos provocados pela problemática da atividade, para que seja possível o desenvolvimento de tais habilidades de forma apropriada.

4 Uso de mecânicas, estratégias e elementos próprios dos jogos, mas aplicados fora do contexto dos games (de Melo Reis et al., 2017).

5 Relaciona-se à aprendizagem "mão na massa", em que o aprendiz combina tecnologias e conhecimentos para a construção de projetos e para a resolução de problemas (Raabe et al., 2017). 
Como trabalhos futuros, o FAE2 foi selecionado para uma validação e apuração deste modelo, baseado na oferta de duas turmas de uma nova formação continuada, realizada durante o primeiro semestre de 2018, em que parte dos resultados são apresentados por Martinelli e Sakata (2018). Outra investigação futura pertinente diz respeito a um estudo mais aprofundado sobre as estratégias de ensino mais propícias para serem aplicadas em práticas que estimulem o Pensamento Computacional nas crianças.

\section{Agradecimentos}

O presente trabalho foi realizado com apoio da Coordenação de Aperfeiçoamento de Pessoal de Nível Superior - Brasil (CAPES) - Código de Financiamento 001. Agradecimentos ao Prof. Dr. Carlos Henrique da Silva Santos (IFSP Câmpus Itapetininga) e às alunas Letícia Mara Berto e Isabella Soares de Lima (C2Y!).

\section{Referências}

BARCELOS, T.; BORTOLETTO, R.; ANDRIOLI, M. Formação online para o desenvolvimento do Pensamento Computacional em professores de Matemática. Anais do Workshop de Ensino em Pensamento Computacional, Algoritmos e Programação, 2016. v. 5. n. 1. p. 1228 - 1237.

BASTOS, N. S.; MOTA, F.; ADAMATTI, D. F.; CARVALHO, F. Developing Computational Thinking in High School: an Approach based on Neuroscience. Journal on Computational Thinking (JCThink), 2017. v. 1 n. 1. p. 28 - 38.

BNCC. Base Nacional Comum Curricular: Educação é a Base. Brasília: Ministério da Educação. Versão Preliminar, 2017. 396 p. Disponível em: <https://goo.gl/xyrJjC >. Acesso em: 19/09/2017.

BRUM, M. G.; KONZEN, A. A.; SCHMACHTENBERG, R. F. Computer Teaching Supported by Gamification. Journal on Computational Thinking (JCThink), 2018. v. 2. n. 1. p. $95-111$.

CHARMAZ, K. A construção da Teoria Fundamentada: Guia Prático para Análise Qualitativa. Bookman Editora, Porto Alegre - RS. 2009. 271 p.

CHOMA, J.; QUINTALE, D.; ZAINA, L. A. M.; BERALDO, D. A perspective-based usability inspection for ERP systems. Proceedings of the 17th International Conference on Enterprise Information Systems (ICEIS'15), 2015. v. 3. p. 57 - 64.

DE MELO REIS, F.; CRISTIANO, F.; MARTINS, D.; DA ROCHA, P. Pensamento Computacional: Uma proposta de ensino com estratégias diversificadas para crianças do Ensino Fundamental. Anais do Workshop de Informática na Escola (XXIII WIE), 2017. v. 23. n. 1. p. $638-647$.

FERREIRA, A. C. C.; MELHOR, A.; BARRETO, J. S.; DE PAIVA, L. F.; MATOS, E. Experiência prática interdisciplinar do Raciocínio Computacional em atividades de computação desplugada na Educação Básica. Anais do Workshop de Informática na Escola (XXI WIE), 2015. v. 21. n. 1. p. 256 - 265.

FRANÇA, R.; TEDESCO, P. Desafios e oportunidades ao ensino do Pensamento Computacional na Educação Básica no Brasil. Anais do Workshop de Ensino em Pensamento Computacional, Algoritmos e Programação, 2015. v. 4. n. 1. p. 1464 - 1473. 
KALELIOGLU, F.; GÜLBAHAR, Y.; KUKUL, V. A framework for Computational Thinking based on a systematic research review. Baltic Journal of Modern Computing, 2016. v. 4. n. 3. p. 583 - 596.

LAZAR, J.; FENG, J. H.; HOCHHEISER, H. Research Methods in Human-Computer Interaction. Wiley. 2010. $426 \mathrm{p}$.

LEITE, M.; DA SILVA, S. F. Redimensionamento da computação em processo de ensino na educação básica: O pensamento computacional, o universo e a cultura digital. Anais do Workshop da Licenciatura em Computação (XXV WEI), 2017. v. 6. n. 1. p. $804-813$.

LIKERT, R. A Technique for the Measurement of Attitudes. Archives of Psychology. American Psychological Association, 1932.

MARTINELLI, S. R.; SAKATA, T. C. (2018). A disseminação do Pensamento Computacional por docentes do Ensino Fundamental I: Relatos de Experiências e Discussões. Anais do Workshop de Informática na Escola (XXIV WIE), 2018. v. 24. n. 1. p. 235 - 244.

MARTINELLI, S. R.; ZAINA, L. A. M.; SAKATA, T. C. O desenvolvimento do Pensamento Computacional em crianças por meio de Atividades de Ensino mediadas pelo Professor do Ensino Fundamental I: Um Estudo de Caso. Universidade Federal de São Carlos (UFSCar Sorocaba), 2018. Relatório Técnico n 004/2018.

RAABE, A.; METZGER, J.; GOMES, E.; VIEIRA, M. V.; SANTANA, A. L. M.; SOUZA, F. T.; RAMOS, G.; CUCCO, L. Características do Pensamento Computacional desenvolvidas em aprendizes do Ensino Médio por meio de atividades makers. Anais do Workshop de Informática na Escola (XXIII WIE), 2017. v. 23. n. 1. p. 145 - 154.

SBC. Computação na Educação Básica. XXXVII Congresso da Sociedade Brasileira de Computação. Versão Preliminar, 2017. 10 p. Disponível em: <https://goo.gl/NNdUv9>. Acesso em: 20/07/2017.

SILVA, V.; DA SILVA, L. L.; FRANÇA, R. Pensamento Computacional na Formação de Professores: experiências e desafios encontrados no ensino da Computação em escolas públicas. Anais do Workshop de Informática na Escola (XXIII WIE), 2017. v. 23. n. 1. p. $805-814$.

VALENTE, J. A. Integração do Pensamento Computacional no currículo da Educação Básica: Diferentes estratégias usadas e questões de formação de professores e avaliação do aluno. Revista e-Curriculum, 2016. v. 14. n. 3. p. 864 - 897.

WING, J. M. Computational Thinking. Communications of the ACM, 2006. v. 49. n. 3. p. $33-35$.

WING, J. M. Computational Thinking Benefits Society. 40th Anniversary Blog of Social Issues in Computing, 2014. Disponível em: <https://bit.ly/2TsgYYs>. Acesso em: $10 / 10 / 2018$ 